\section{In der Psychosomatik kann man GOÄ-Ziffern oft kombinieren}

\begin{abstract}
_ In der GOÄ steht für die Behandlung eines Patienten mit psychosomatischen oder neurotischen Störungen die Nr. 849 zur Verfügung. Die Leistung wird im Schwellensteigerungssatz von $2,3 \mathrm{mit}$ 30,84 Euro vergütet. Die Sitzung muss mindestens 20 Minuten dauern. Eine Differenzierung nach Diagnostik und Therapie wie im EBM (Nrn. 35100 und 35110) ist nicht vorgesehen. Allerdings
\end{abstract}

kommt für eine eingehende psychiatrische Untersuchung, ggf. mit Kontakt zur Bezugsperson, die Nr. 801 GOÄ infrage. Sie wird im Schwellensteigerungssatz mit 33,51 Euro vergütet.

\section{MMW-KOMMENTAR}

Wenn die Leistungen erbracht sind, können die Nrn. 801 und 849 GOÄ auch in einer Sitzung kombiniert abgerechnet werden. Auch

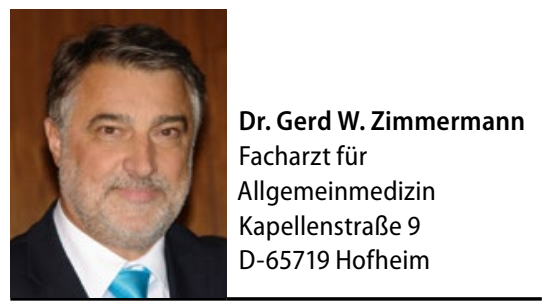

eine zur Abgrenzung erforderliche neurologische Untersuchung nach Nr. 800 oder eine somatische Abklärung nach den Nrn. 5-7 wären abhängig vom Einzelfall im Rahmen des gleichen Kontakts abrechenbar. Beachtenswert ist, dass insbesondere der Leistungsinhalt der Nr. 849 keinesfalls nur einem bestimmten Fachbereich zugeordnet ist, sondern von allen Fachgruppen erbracht werden kann.

Beihilfestellen verweigern Hausarztpraxen allerdings oft die Bezahlung, da dort im Rahmen der psychosomatischen Grundversorgung ohne vertrauensärztliches Gutachten nur zehn Sitzungen je Krankheitsfall beihilfefähig sind. Das Verwaltungsgericht Oldenburg bestätigte dies 2002. Die Richter wollten verhindern, dass die beihilferechtlichen $\mathrm{Be}$ schränkungen bei Psychotherapien umgangen werden (Az.: 6 A 3926/99).

Natürlich betrifft diese Einschränkung den Versicherten, der die Behandlung nicht erstattet bekommt. Der Arzt ist eigentlich nicht tangiert - sollte den Patienten aber darauf hinweisen, um Streitereien zu vermeiden.

\title{
Spezialisierte Geriatrie bekommt MVZ-Klausel
}

_ Die Präambel zum neuen EBM-Abschnitt 30.13 über die spezialisierte geriatrische Diagnostik und Versorgung regelt zurzeit, dass die Nr. 30984 für ein weiterführendes geriatrisches Assessment nur berechnet werden kann, wenn die Leistung auf Überweisung eines Vertragsarztes nach Nr. 1 derselben Präambel erfolgt. Dies kann ein Hausarzt oder im Ausnahmefall auch ein Neurologe oder Psychiater sein, und es muss eine Vorabklärung nach den Nrn. 30980 und 30981 stattgefunden haben. Erstere wird dabei von dem überweisenden Arzt abgerechnet, letztere von dem spezialisierten Geriater oder einer geriatrischen Ambulanz nach Nr. 2 der Präambel.

\section{MMW-KOMMENTAR}

Bisher gab es ein Problem, wenn der Vertragsarzt nach Nr. 1, etwa ein Neurologe, und der Vertragsarzt nach Nr. 2, etwa ein Hausarzt mit der Zusatzbezeichnung Geriatrie, in derselben Berufsausübungsgemeinschaft (BAG) oder demselben Medizinischen Versorgungszentrum (MVZ) arbeiteten. Innerhalb dieser Kooperationsformen sind nämlich keine Überweisungen erlaubt - der Nr. 30984 fehlten also die Voraussetzungen. Durch eine Ergänzung der Präambel soll deshalb klargestellt werden, dass in BAG und MVZ auf eine Überweisung verzichtet werden kann, sofern sich die Notwendigkeit für ein weiterführendes geriatrisches Assessment aus einem hausärztlich-geriatrischen Basisassessment nach Nr. 03360 ergibt. Eine Vorabklärung zwischen den beiden Ärzten soll in diesem Fall aber nicht gesondert über die Nrn. 30980 und 30981 vergütet werden. 